\title{
Insight into Audit Reports on the Causes of Maternal Deaths in Poor Health Settings: The Case of North Kivu Province in Demo- cratic Republic of Congo
}

\author{
Woolf Kapiteni ${ }^{1}$, Célestin Mamba ${ }^{2}$ and Justin N Kadima ${ }^{3 *}$ \\ ${ }^{1}$ Department of Nursing Sciences, Medical Technical Institute of Higher Education (ISTM Kiroshe), \\ Democratic Republic of the Congo \\ ${ }^{2}$ Department of Nursing Sciences, Medical Technical Institute of Higher Education (ISTM Luebo), \\ Democratic Republic of the Congo \\ ${ }^{3}$ Department of Clinical Pharmacology, School of Medicine \& Pharmacy, University of Rwanda, Rwanda
}

*Corresponding author: NJ Kadima, Department of Clinical Pharmacology, School of Medicine \& Pharmacy, University of Rwanda, Rwanda

\begin{abstract}
Background: Maternal mortality is a major public health problem in the world and particularly in developing countries. Understanding the causes and responsibilities remain capital when action is needed by policymakers and practitioners or by caregivers. The objective was to analyze audit data reported on maternal deaths and highlight the major causes of mortality as well as responsibilities.

Methods: We analyzed 48 cases of maternal deaths that were reported to the health information bureau of the province of North Kivu in DR Congo in the first semester of 2017. The audit was conducted in various health facilities located in both rural and urban areas including General Reference Hospital and Reference Health Centers as Category-2, private polyclinics and small medical centers as Category-1. The audit consisted of verbal autopsy, verbal determination of non-clinical causes of death and verbal communication of basic characteristics using a standardized national Audit Form. The causes of mother death were defined as direct or indirect and related to heath facilities or to mother next of kin.

Results: The analysis shows the mothers were aged 15-43 years, of which $54.9 \%$ were in good health at the admission to health centers. The causes were considered as direct in $75.1 \%$ and indirect in $24.9 \%$ of all cases. More than half of women died of hemorrhage (52\%) followed by thromboembolism and cardiovascular diseases (14.6\%). Lack of quality infrastructure in health facilities and incompetence of care practitioners were responsible for $68 \%$ of deaths against $32 \%$ cases related to mothers and next of kin persons. The majority of deaths occurred in multiparinous (45.8\%) than primiparous $(20.8 \%)$ mothers, during postpartum $(64.5 \%)$ than intrapartum $(6.2 \%)$ period, in rural $(62.5 \%)$ than in urban $(37.5 \%)$ areas.
\end{abstract}

Conclusions: Maternal mortality is subjected to many causes implicating particularly poor quality of infrastructures and inexperience of practitioners. That is, upgrading the infrastructure of obstetrical medical facilities and the professionalism of nurses and other health practitioners, while educating pregnant women about childbirth good practice, may significantly reduce maternal mortality in this Province and in the Country.

\section{Keywords}

Maternal death audit, Causes, Responsibilities, Verbal autopsy, North Kivu DR Congo

\section{Introduction}

Being pregnant is a desire that most women aspire to at some point in their lives. However, pregnancy carries some risk of death [1,2]. Maternal death is the death of a woman during pregnancy or within 42 days of the end of pregnancy, regardless of the duration and location of pregnancy, any cause related or aggravated by pregnancy or management but no accidental or incidental causes [3]. Every 2 minutes in the world, a maternal death can be notified [2]. Each year, more than 350,000 women die from complications related to pregnancy or childbirth [4,5], 99\% of which occur in developing countries. In developing countries, one in 16 women can die from pregnancy-related complications, compared with one in 2800 women in developed countries [1].

\footnotetext{
Citation: Kapiteni W, Mamba C, Kadima JN (2018) Insight into Audit Reports on the Causes of Maternal Deaths in Poor Health Settings: The Case of North Kivu Province in Democratic Republic of Congo. Int J Womens Health Wellness 4:079. doi.org/10.23937/2474-1353/1510079

Accepted: October 15, 2018: Published: October 17, 2018

Copyright: (c) 2018 Kapiteni W, et al. This is an open-access article distributed under the terms of the Creative Commons Attribution License, which permits unrestricted use, distribution, and reproduction in any medium, provided the original author and source are credited.
} 
Globally, maternal mortality has declined significantly between 1990 and 2015, but not rapidly enough to meet the United Nations Sustainable Development Goals (SDGs), a $75 \%$ reduction in the number of live birth deaths in the world by 2030 [6]. The global maternal mortality ratio (number of maternal deaths per 100,000 live births) decreased by $2.3 \%$ per year between 1990 and 2015. In some countries, annual decreases were greater than $5.5 \%$ between 2000 and 2010 [6]. Some countries, however, have made significant improvements through better access to health care and an increasing proportion of girls accessing education. In sub-Saharan Africa, a number of countries have also halved their maternal mortality rates since 1990, and increased rates of accelerated decline have been observed since 2000 [6].

Every maternal death is a huge loss in Africa. The death of a mother is a tragic event that has an impact on the well-being of her family. This implies a loss of income and contributes to the impoverishment of the family in particular and of society in general [7]. The absence of a mother affects the survival and development of children with a tenfold risk of dying and this is unacceptable [8-10]. The maternal mortality rate is an indicator used to monitor the progress of the health system in a community [11]. The United Nations has advocated that women play a vital role in the development of humanity; Investing in health is therefore a way to build a stable, peaceful and productive society [12].

A WHO meta-analysis [13] indicates that about $73 \%$ $(1,771,000$ out of $2,443,000)$ of all maternal deaths between 2003 and 2009 were due to direct obstetric causes and indirect causes accounted for $27.5 \%(672,000$, $95 \%$ UI 19.7-37.5) of all deaths. Hemorrhages accounted for $27.1 \%(661,000,19.9-36.2)$, hypertensive disorders $14.0 \%(343,000,11.1-17.4)$ and sepsis $10.7 \%(261,000$, 5.9-18.6) of maternal deaths. The remaining deaths were due to abortion (7.9\% [193,000], 4.7-13.2), embolism $(3.2 \%[78,000], 1.8-5.5)$, and all other direct causes of death $(9.6 \%[235,000], 6.5-14.3)$. Regional estimates varied considerably.
Currently, the causes of maternal mortality are preventable in most cases. The quality of care provided plays an important role in the prevention and management of pregnancy complications, as it can reduce the number of maternal deaths $[14,15]$. Understanding the causes and responsibilities therefore remains crucial when policy makers and practitioners need to take action. The evaluation of the performance of a health system can be carried out through the audit. The audit is a systematic and critical analysis of the quality of medical care, a compilation of collected data on the procedures of diagnosis, treatment, use of resources, results, quality of life, risk factors, preventable factors contributing to death, health facility, as well as the community [1619].

As summarized in an article by Wijeratne $D$ and Weeks AD [20], it is estimated that the civil war in DRC has killed 4 million people since 1998 and has been fueled to a large extent by competition for the country's natural resources [21]. It is also estimated that mineral resources in the DRC are worth $f 15$ trillion, more than the combined GDP of Europe and the United States [22]. However, in 2013, despite this considerable potential wealth, the DRC was ranked $186^{\text {th }}$ out of 187 countries in the UN Human Development Index, which ranks countries on the basis of a composite statistic of life expectancy, education and indices of income inequality [23]. At the height of the civil war between 1998 and 2003, about 98 percent of the mining industry in the DRC was forced to work under the control of various militia groups [24]. The main objects of expropriation were gold and minerals such as Casserite (tin ore), Wolframite (tungsten ore) and Coltan (tantalum ore), essential for the manufacture of consumer electronics products such as mobile phones. Hitherto, those militia groups continue to commit disasters by killing, raping women including adolescents. One study reported an incidence of 48 rapes every hour in eastern DRC [25].

At the peak of the civil conflict, the maternal mortality rate in the DRC was more than 1,100 per 1,000,000. Due to the marked reduction in the availability of financial capital to fuel the ongoing conflict, increasing-
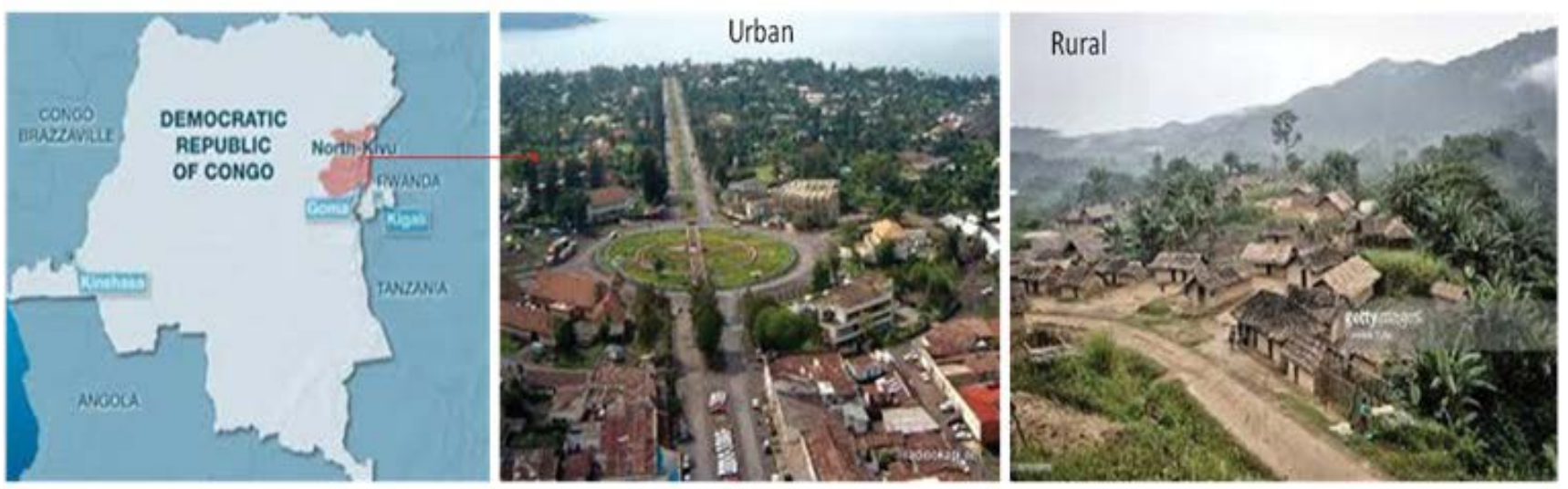

Figure 1: North Kivu in earsten DR Congo, urban and rural areas (Google). 
ly important resources are diverted to health care and other areas of society's development. With regard to maternal health, the focus is again on the provision of skilled birth attendants, emergency obstetric care and improved access to contraception [26]. In 2015, the maternal mortality rate (maternal deaths per 100,000 live births) was 696 in the DRC, compared to 546 in sub-Saharan Africa, 216 in the world and 25 in ECA - Europe and Central Asia $[6,19]$. This figure still represents one of the highest maternal mortality rates in the world and is subject to some uncertainty because of the difficulties in accurately measuring mortality in areas without formal registration systems. Eastern DRC has the highest rate of sexual violence in the world, with rape frequently used as a weapon of war. The purpose of this study was to highlight the causes of maternal mortality and responsibilities in the North Kivu province in the DRC using audit reports (Figure 1).

\section{Methods}

\section{Study area}

North Kivu (French: Nord Kivu) is an area of 59483 square kilometers located in eastern DRC, bordering Lake Kivu, with about 6 million inhabitants (39 people/ $\mathrm{km}^{2}$ ), 60\% live in rural areas. The province is home to Virunga National Park, World Heritage Site containing endangered mountain gorillas. The province comprises three cities (Goma, Butembo and Beni) and six territories (Beni, Lubero, Masisi, Rutshuru, Nyiragongo and Walikale). Goma is the capital. The region is politically unstable and has been one of the starting points for military conflicts in the region since 1998. The population lives mainly from agriculture and small businesses in goods trading and mining.

\section{Health facilities}

According to the structure of the national health care system $[27,28]$, the DRC country has been subdivided since 1985 into health zones (HZ) and each $\mathrm{HZ}$ subdivided into health area (HA). $\mathrm{HZ}$ covers about 100,000 inhabitants (rural) or 150,000 to 200,000 inhabitants (urban). For proper functioning, each $\mathrm{HZ}$ must have a general reference hospital (GRH) and also one or more reference health centers (RHC) depending on the demographic size of its population. Other categories of health structures include private clinics, polyclinics, and medical centers. For simplicity, health facilities are divided into category-1 (small size settings) and category-2 (reference hospitals or big centers). In many rural health centers, nurses and midwives play a vital role in managing pregnancy problems.

\section{Audit procedure}

The audit of maternal death (MDA) is a post-mortem care and includes three approaches: verbal autopsy (VA), verbal determination of non-clinical causes of death (VDNCC) and verbal communication of basic char- acteristics (VCBC) [29-32]. VA is an interview with relatives (neighbors) of the deceased to reconstruct events before death to obtain a clinically accepted obstetrical/ medical diagnosis. VDNCC is a re-enactment of factors associated with seeking care behavior and accessing and delivering services. VCBC reports on age, education and other social variables. An analogy has been established with the "confidential investigation" or the "maternal death check" practiced in the United Kingdom (United Kingdom). A confidential survey, as understood in the United Kingdom, consists of an audit of health care providers and is initiated by public health authorities to gather information on the circumstances that led to a maternal death [29-32]. The audit of providers and care (APC) consists of a review of medical records, laboratory results, prescriptions, X-rays and other data to determine obstetric/medical causes of death and to determine non-medical factors contributing to death. The investigation reports were reviewed by independent reviewers who added their comments and opinions regarding the cause(s) of death [29].

\section{Data collection}

At the facility level, data were obtained through routine registration of deaths when that occurs, meaning as usual practice. In the event of death, the care team completes a death registration form, lists the conditions or illnesses leading to death, and assigns a condition as the underlying cause of death according to their experience. A standard national reporting form established by the Ministry of health and adapted from WHO tool [32] is used. The exercise is accomplished by a qualified medical team. For maternal mortality, the end point is death during pregnancy or within 42 days of the end of pregnancy. In situations where deaths occurred at home in the absence of qualified medical personnel, relatives of the deceased were also asked about the circumstances that led to the death. The data were collected by qualified physicians or epidemiologists investigators working with the Ministry of Health. They should be more inclined to record responses as reported by respondents in the local language. It was obvious that the investigator had to decide who to interview.

\section{Study sample}

The sample consisted of available 48 maternal deaths reported in the first half of 2017 to the Central Health Information Office of the Province.

\section{Data analysis}

The analysis was performed using the Excel 2016 software to calculate frequencies, proportions, or ratios. The primary endpoint was pregnancy-related death. Causes of death were classified as direct or indirect. Health facilities causes were related to lack of equipment, poor infrastructure, dysfunction, lack of medication or incompetence of practitioners. Mother related causes were late or no prenatal consultation, sociodemographic fac- 
Table 1: Distribution by Demographics (cases $=48$ ).

\begin{tabular}{|c|c|c|c|c|c|c|c|}
\hline Variable & $\mathbf{N}$ & $\%$ & $\mathbf{R}$ & Variable & $\mathbf{N}$ & $\%$ & $\mathbf{R}$ \\
\hline Age & & & & Marital status & & & \\
\hline $15-29$ years & 22 & 45.8 & 0.84 & Married & 22 & 45.8 & 0.84 \\
\hline $30-49$ years & 26 & 54.2 & 1 & Others & 26 & 54.2 & 1 \\
\hline Parity & & & & Antenatal visits & & & \\
\hline 1 child & 10 & 20.8 & 0.45 & Yes & 36 & 75.0 & 1 \\
\hline 2-4 children & 16 & 33.3 & 0.72 & No & 8 & 16.7 & 0.22 \\
\hline$>4$ children & 22 & 45.8 & 1 & Missing & 4 & 8.3 & 0.11 \\
\hline Education & & & & Profession & & & \\
\hline Illiterate & 11 & 22.9 & 1 & Farmer & 24 & 50.0 & 1 \\
\hline Primary & 9 & 18.8 & 0.82 & Housewife & 15 & 31.3 & 0.62 \\
\hline Secondary & 9 & 18.8 & 0.82 & Student & 1 & 2.1 & 0.04 \\
\hline University & 2 & 4.2 & 0.18 & Nurse & 1 & 2.1 & 0.04 \\
\hline Missing & 17 & 35.4 & & Others & 7 & 14.7 & 0.29 \\
\hline
\end{tabular}

Table 2: Direct and indirect causes of maternal deaths.

\begin{tabular}{|l|l|l|l|l|l|}
\hline Direct causes & N & \% & Indirect causes & N & \% \\
\hline Hemorrhage & 25 & 52.1 & Malaria & 3 & 6.3 \\
\hline Thromboembolism & 3 & 6.3 & Traditional remedies & 2 & 4.2 \\
\hline Hypertensive crisis & 2 & 4.2 & Cardiovascular disease & 2 & 4.2 \\
\hline Dystocic childbirth & 2 & 4.2 & Anemia & 2 & 4.2 \\
\hline Infection sepsis & 2 & 4.2 & HIVIAIDS & 1 & 2.1 \\
\hline Complication to abortion & 1 & 2.1 & Difficult to evaluate & 2 & 4.2 \\
\hline Allergy to anesthesia & 1 & 2.1 & & & 2 \\
\hline s/Total & 36 & 75.1 & s/Total & 24.9 \\
\hline
\end{tabular}

Table 3: Responsibilities of Heath facilities and Mother next of kin.

\begin{tabular}{|c|c|c|c|c|c|}
\hline Heath facilities & $\mathbf{N}$ & $\%$ & Parturients next of kin & $\mathbf{N}$ & $\%$ \\
\hline Inappropriate care & 24 & 50.0 & Delay in decision taking & 19 & 39.6 \\
\hline Inappropriate follow up & 17 & 35.4 & Delayed transfer to appropriate $\mathrm{HC}$ & 16 & 33.3 \\
\hline Delay in decision making & 14 & 29.2 & Close pregnancies & 11 & 22.9 \\
\hline Delivery plan not respected & 10 & 20.8 & Multiparous & 6 & 12.5 \\
\hline Lack of equipment & 8 & 16.7 & Ignorance & 6 & 12.5 \\
\hline Lack of blood donors & 7 & 14.6 & Refusal to transfer mother to hospital & 5 & 10.4 \\
\hline Lack of medicines & 7 & 14.6 & Self-medication & 4 & 8.3 \\
\hline Lack of competent staff & 5 & 10.4 & Absence of prenatal consultation & 1 & 2.1 \\
\hline Problem of transport between $\mathrm{HZ}$ & 5 & 10.4 & Late prenatal consultation & 1 & 2.1 \\
\hline Lack of communication between $\mathrm{HC}$ & 2 & 4.2 & Minor primiparous & 1 & 2.1 \\
\hline Absence of practitioner & 1 & 2.1 & & & \\
\hline Inattention & 1 & 2.1 & & & \\
\hline Non-authorized structure & 1 & 2.1 & & & \\
\hline Non-reference to appropriate centers & 1 & 2.1 & & & \\
\hline
\end{tabular}

tors, health status upon admission. The time of death (prenatal, intrapartum, postpartum) and complications were determined. Late deaths that could occur 42 days after hospital discharge were not included.

\section{Results}

\section{Demographic and reproductive profile of parturient}

Table 1 describes the demographic and reproductive characteristics of the 48 deceased mothers. The average age was 29 (15-43), with $45.8 \%$ between 15 and 30-yearsold and $54.2 \%$ between 30 and 49 -years-old; $45.8 \%$ were married; $45.8 \%$ were multiparinous $>4$ children, $33.3 \%$ multiparinous from 2 to 4 children and $20.8 \%$ primiparous; $75 \%$ had an antenatal consultation; $22.9 \%$ were illiterate and only $4.2 \%$ had a university level; the majority were farmers (50\%) and housewives (31\%).

\section{Causes of maternal deaths and responsibilities}

Table 2 direct causes accounted for 36 (75.1\%) deaths versus 12 (24.9\%) for indirect causes. Among direct causes, hemorrhages accounted for $52.1 \%$. Indirect causes included anemia, heart disease, malaria, HIV/ AIDS and intoxication with traditional treatment. The classification has been adapted from the WHO classification of direct and indirect causes of maternal death.

Table 3 inadequate actions attributable to health facilities consisted of inappropriate care (50\%), inadequate follow-up (35.4\%), delayed decision-making (29.2\%), delivery plan not respected $(20.8 \%)$, lack of equipment (14.6\%), lack of medication (14.6\%), lack of blood donors (14.6\%), absence of competent staff $(10.4 \%)$, etc. The main causes related to parturients 
Table 4: Effect of Location and Category of Health facilities.

\begin{tabular}{|c|c|c|c|c|c|c|}
\hline \multirow[b]{2}{*}{ Location } & \multicolumn{2}{|c|}{ Category-1 } & \multicolumn{2}{|c|}{ Category-2 } & \multicolumn{2}{|c|}{ Total } \\
\hline & $\mathrm{N}$ & $\%$ & $\mathrm{~N}$ & $\%$ & $\mathrm{~N}$ & $\%$ \\
\hline Rural & 6 & 12.5 & 24 & 50 & 30 & 62.5 \\
\hline Urban & 3 & 6.3 & 15 & 31.3 & 18 & 37.5 \\
\hline Total & 9 & 18.8 & 39 & 81.2 & 48 & 100 \\
\hline
\end{tabular}

Table 5: Period of death vs. mother's health at admission.

\begin{tabular}{|l|l|l|l|l|l|l|l|}
\hline Time of death & \multicolumn{3}{|l|}{ Good health } & \multicolumn{3}{|l|}{ Bad health } & \multicolumn{2}{l|}{ Total } \\
\hline & $\mathrm{N}$ & $\%$ & $\mathrm{~N}$ & $\%$ & $\mathrm{~N}$ & $\%$ \\
\hline Abortion & 0 & 0 & 1 & 2.1 & 1 & 2.1 \\
\hline Prenatal & 2 & 4.2 & 3 & 6.3 & 5 & 10.4 \\
\hline Intrapartum & 3 & 6.3 & 0 & 0 & 3 & 6.3 \\
\hline Postpartum & 21 & 43.8 & 10 & 20.8 & 31 & 64.6 \\
\hline Total valid & 26 & 54.2 & 14 & 29.2 & 40 & 83.3 \\
\hline
\end{tabular}

Missing $=8(16.7 \%)$ reports.

were delay in decision taking (39.6\%), late attendance to an appropriate health center (33.3\%), close pregnancies $(22.9 \%)$, Multiparous (12.5\%), ignorance (12.5\%); refusal to transfer mother to hospital (10.4\%), self-medication (8.3\%), etc. One case of self-medication and the 5 who refused to go to hospital died at home.

Table 4 the impact of location and type of the health facility shows $62.5 \%$ of deaths occurred in rural health facilities compared to $37.5 \%$ in urban health facilities; $81.3 \%$ of deaths were in Category 2 health facilities compared to $18.8 \%$ in Category 1.

Table 5 the impact of the health status of mother before admission to the obstetric service shows 26 (54.2\%) mothers arrived in good physical condition compared with 14 (29.2\%) classified clinically in poor condition on admission (there was no information in 8 (16.7\%). Among those in good health, 21 (43.8\%) died in postpartum, $3(6.3 \%)$ in intrapartum and $2(4.2 \%)$ in prenatal meantime, and no case of abortion. Among 14 mothers in bad health, $10(20.8 \%)$ died in postpartum, zero in intrapartum, $3(6.3 \%)$ in prenatal and 1 (2.1\%) abortion. The prenatal period for human females usually lasts about nine to ten months or forty weeks on average, even up to forty-two weeks if safely; Intrapartum is the period between the beginning of work and the end of the third stage of labor, while the postpartum is the period after delivery.

\section{Discussion}

The study aimed to highlight the causes and responsibilities of maternal deaths in North Kivu province in DRC based on audit reports. The demographic profile of parturients showed that maternal mortality can occur in any women of childbearing age, married or single. However, the risk would be higher for uneducated and multiparous mothers. High proportions of farmers and housewives found among deaths correlate with the fact that the majority of people in North Kivu practice agriculture and many are unemployed.

The report pointed out that $56.3 \%$ of parturients concerned had antenatal consultation (ANC). However, only $10-12 \%$ of women died in prenatal period among $43.7 \%$ who did not go ANC. In general, the impact of ANC on saving lives of pregnant women at the admission time is minimal here due to high impact of other factors. A study conducted in Kinshasa the capital of the DRC, showed that of all the women who died in the study sample, $46 \%$ of women did ANC [33].

This study showed that direct causes accounted for $75.1 \%$ of which $52.1 \%$ as hemorrhages and indirect causes for $24.9 \%$. Other direct causes were embolism (6.2\%), hypertensive crisis (4.2\%), dystocia (4.2\%), sepsis (4.2\%), and complication of abortion (2.1\%). Indirect causes consisted of anemia, heart disease, malaria, HIV/ AIDS and intoxication by traditional treatment. This is consistent with what has been reported in different WHO regions between 2003 and 2009 showing 73\% of direct obstetric causes and $27 \%$ of indirect causes [13]. In sub-Saharan Africa, the main causes reported were hemorrhages $24.5 \%$ (16.9-34.1), hypertension $16 \%$ (11.7-21), abortion 9.6\% (5.1-17.2), embolism 2.1\% (0.8-4.5), sepsis 10.3\% (5.5-18.5) [26]. Dellagi, et al. [34] found $69.8 \%$ hemorrhage. Touaibia, et al. [35] described the most common causes of death as bleeding (75.6\%), hypertensive complications (28\%), dystocia (12\%), heart disease (6\%) and infections (4\%). One study [36] conducted in the city of Lubumbashi (DRC) at Sendwe hospital indicates that out of 77 maternal deaths were identified during 2013-2015, $74.03 \%$ of deaths occurred direct obstetric causes; bleeding with $61.04 \%$ was the leading cause of maternal death followed by eclampsia $(31.58 \%)$; indirect causes were dominated by heart disease $(30.0 \%) ; 75.32 \%$ of deaths had occurred within 24 hours of admission. The major causes are similar but occurring with different rates according to areas of studies.

Responsibilities can be shared by both the health centers and the mothers. The number of deaths in rural area (62.5\%) was higher than the number in urban area (37.5\%). This is also consistent with the study by Chaibou, et al. [37] that found $76.1 \%$ of deaths in rural areas. However, there were more deaths in Category-2 of health facilities compared to Category- 1 . That is explained by the fact that this category includes reference hospitals where serious cases are transferred to.

In this study, $54 \%$ of parturients were in good health and $28 \%$ in poor health when they have been admitted at the obstetrical services. The state of health of the mother may be an aggravating factor of risk. Since the mothers died from hemorrhages that occurred mainly in postpartum, the poor condition of the mother at admission may certainly be a risk factor, but easily manageable in well equipped heath settings.

With respect to health facilities, the responsibilities are related to inadequate infrastructure and lack of qualified people in rural and small health facilities, con- 
sistent with the study of Hynes, et al. [38]. According to that study, the authors showed that $52.1 \%$ of the caregivers had received training in normal (uncomplicated) deliveries, while only 10 (43.4\%) had received training on the complications of childbirth. Mouzou, et al. [39] found in their study that almost all deceased parturients who underwent caesarean had not benefited from the physician's services for resuscitation.

With respect to mothers, the responsibilities are related to culture, poverty, negligence and ignorance. These are characterized by delay or refusal in taking decision to attend health centers, close pregnancies, self-medication and absence of ANC. This has also been noticed in Kinshasa [40]. In general, lack of money and transportation or ignorance would prevent poor families from accessing obstetric centers quickly. The study of Hynes, et al. [38] reported that about $80 \%$ of the women arrived at the health center on foot and $7 \%$ of women had had a pregnancy at home. We agree with Alexander Dumont [14] who concluded that the delay in care has a fatal outcome for the mother in developing countries. Maternal death is an indicator of the weaknesses of the health system and development as well in a country [23]. However surprisingly, even in the United States, the number of maternal deaths remains high. A health system can be strong but the cost of care must not be out of reach of the population [41].

Many cases of maternal deaths are too often solitary or hidden events and go uncounted because of the inherent weakness of health information and records, particularly in countries where non medical abortion is prohibited. Also "near misses" defined as women who almost died but, with luck and care, survived [42] were not accounted in the present study, contrarily to what is done in developed countries where maternal deaths are rare.

\section{Conclusions}

The study found that a large proportion of maternal deaths were due to delayed or insufficient emergency obstetric care, inadequate management, lack of medical and technical equipment. Thus, improving the infrastructure of obstetric medical facilities and the professionalism of health practitioners, while educating pregnant women on good birthing practices and family planning can significantly reduce maternal mortality in that province. There is an urgent need to develop and validate emergency interventions designed to improve the management of obstetric complications in resource-poor settings and implement friendly sexual and reproductive services.

\section{Declarations}

\section{Acknowledgements}

The authors would like to acknowledge the Provincial Bureau of Health Information for accepting to avail audit reports.

\section{Funding}

No financial support was received in conducting this study.

\section{Availability of data and materials}

Data presented in this paper and French version of the questionnaire are available from the corresponding author on reasonable request.

\section{Authors' contributions}

$\mathrm{KW}$ and MC developed the protocol, collected and analyzed data and wrote the first draft. KNJ validated the protocol, revised data analysis and wrote the final draft. All authors read and approved the final version.

\section{Ethics approval and consent to participate}

In this study, the anonymity and confidentiality of the information was guaranteed. The study was cleared by the ethical committee of Medical Technical Institute of Higher Education (ISTM Kirotshe), DR Congo. Informed consent was obtained from next of kin persons to be audited and they were informed the results could be used for scientific purposes. For teens under 17 years, the parents consented verbally to interact with auditors. Culturally, the consent is almost verbal as the majority is illiterate. There was no more need to obtain consent for analyzing audit reports.

\section{Competing interests}

The authors declare that they have no competing interests.

\section{Consent for publication}

Not applicable.

\section{Authors' information}

$\mathrm{KW}$ is $\mathrm{MD}, \mathrm{MPH}$ and lecturer of gynecology. $\mathrm{MC}$ is also $\mathrm{MD}, \mathrm{MPH}$ and lecturer in nursing. KNJ is a Professor of pharmacology, clinical therapeutic and family planning.

\section{References}

1. WHO (2004) Beyond the numbers: Reviewing maternal deaths and complications to make pregnancy safer.

2. WHO (2015) Maternal mortality. Global Health Observatory (GHO).

3. WHO (2016) Maternal mortality.

4. WHO (2015) Levels and trends in child mortality: Report 2015. Inter-Agency Group of United Nations on the evaluation of the child's mortality.

5. WHO/UNICEF/UNFP (2012) Tendencies of the maternal mortality: 1990-2010.

6. Alkema L, Chou D, Hogan D, Zhang S, Moller AB, et al. (2016) Global, regional, and national levels and trends in maternal mortality between 1990 and 2015, with scenario-based projections to 2030: A systematic analysis by the in Maternal Mortality Estimation Inter-Agency Group. Lancet 387: 462-474. 
7. WHO (2017) Global Strategy for Women's and Children's health.

8. WHO (2000) Reducing maternal mortality: A challenge for the $21^{\text {st }}$ century.

9. WHO (2013) Maternal death surveillance and response: Technical guidance. Information for action to prevent maternal death.

10. de Brouwere V (2017) Humanitarian programme \& development. Maternal mortality reduction in developing countries: A challenge of the society.

11. Van den Broek NR, White SA, Ntonya C, Ngwale M, Cullinan TR, et al. (2003) Reproductive health in rural Malawi: A population-based survey. BJOG 110: 902-908.

12. Dellagi RT, Bougatef S, Ben Salah F, Ben Mansour N, Gzara A, et al. (2014) National survey of maternal mortality of 2010: Data of Tunisia. Tunis Med 92: 560-566.

13. Say L, Chou D, Gemmill A, Tunçalp Ö, Moller AB, et al. (2014) Global causes of maternal death: A WHO systematic analysis. Lancet Glob Health 2: e323-e333.

14. Dumont A (2012) How Can Maternal Mortality be reduce? Bull Acad Natle Méd 196: 1521-1534.

15. Bhutta ZA, Das JK, Bahl R, Lawn JE, Salam RA, et al. (2014) Can available interventions end preventable deaths in mothers, newborn babies, and stillbirths, and at what cost? Lancet 384: 347-370.

16. Denison DM (1995) The audit handbook: Improving health care through clinical audit. Qual Health Care 4: 68-69.

17. Zanconato G, Machungo F, Soler A, Bergstrom S (1994) Audit of uterine rupture in Maputo: $A$ tool for assessment of obstetric care. Gynecol Obstet Invest 38: 151-156.

18. Bullough C, Graham W (2000) Criterion-based audit of maternal deaths and life threatening complications. In: Qualitative approaches for investigating maternal deaths. Geneva: World Health Organization 2000.

19. Bergstrom S, Bugalho A (1992) Perinatal audit of one hundred consecutive vacuum extractions in Maputo. Gynecol Obstet Invest 34: 171-174.

20. Dileep Wijeratne, Andrew David Weeks (2017) Reducing maternal mortality in sub-Saharan Africa: The role of ethical consumerism. J Glob Health 7: 010309.

21. Coghlan B, Brennan RJ, Ngoy $P$, Dfoy $D$, Otto $B$, et al. (2006) Mortality in the democratic republic of Congo: A nationwide survey. Lancet 367: 44-51.

22. United Nations Environment Program (2011) The Democratic Republic of Congo: Post-Conflict Environmental Assessment, Synthesis for Policy Makers.

23. United Nations Development Program (2013) Rise of the South: Human Progress in a Diverse World. The $2013 \mathrm{Hu}$ man Development Report.

24. World Health Organisation (2015) Trends in maternal mortality: 1990 to 2015 . Estimates by WHO, UNICEF, UNFPA, World Bank Group and the United Nations Population Division.

25. Peterman A, Palermo T, Bredenkamp C (2011) Estimates and determinants of sexual violence against women in the Democratic Republic of Congo. Am J Public Health 101: 1060-1067.

26. World Health Organisation (2014) Strategies toward ending preventable maternal mortality (EPMM).
27. UNFPA (2017) End line evaluation of the H4+ Joint Programme Canada and Sweden (Sida) 2011-2016.

28. (2006) Stratégie de renforcement du système de santé. Kinshasa (DRC): Ministère de la Santé Publique de la République Démocratique du Congo.

29. WHO (1994) Verbal autopsies for maternal deaths.

30. Smith $\mathrm{H}$, Ameh $\mathrm{C}$, Roos N, Mathai M, van den Broek N (2017) Implementing maternal death surveillance and response: A review of lessons from country case studies. BMC Pregnancy Childbirth 17: 233.

31. Iyer A, Sen G, Sreevathsa A (2013) Deciphering rashomon: An approach to verbal autopsies of maternal deaths. Global Public Health 8: 389-404.

32. (2011) Maternal death audit as a tool reducing maternal mortality.

33. Kayembe C (2001) RD Congo: Etude auprès des infrastructures sanitaires des facteurs à la base de la mortalité à Kinshasa. UN Office for the Coordination of Humanitarian Affairs (OCHA).

34. Dellagi RT, Belgacem I, Hamrouni M, Zouari B (2008) Evaluation of the maternal death system in public facilities in the Tunis (1999-2004). Eastern Mediterranean Health Journal 14: $1380-1390$.

35. Touaibia S, Youcef E, Zahra (2017) Prevalence of the maternal mortality in the region of Chief.

36. Odette KM, Moise KK, Blood BND, Mukendi CP, Réne JMM, et al. (2017) Etiologies of maternal mortality in the hospital provincial janson sendwe in Lubumbashi (DR. Congo). Open Access Library Journal 4: 1-8.

37. Chaibou MS, Kaboré RAF, Zoumenou E, Oumarou I, Djibril B, et al. (2013) Mortality and morbidity kindergartens in obstetric anesthesia for Caesarean. RAMUR (Special convention) 18: 8 .

38. Hynes M, Meehan K, Meyers J, Maneno LM, Hulland E (2017) Using a quality improvement approach to improve maternal and neonatal care in North Kivu, Democratic Republic of Congo. Reprod Health Matters 25: 140-150.

39. Mouzou T, Tomta K, Egbohou P, Fiagnon K, Sama H, et al. (2013) Maternal death in gynecological and obstetric resuscitation at university hospital centre Sylvanus Olympio of Lomé. RAMUR (Special convention) 18: 21.

40. Kabali E, Gourbin C, De Brouwere V (2011) Complications of childbirth and maternal deaths in Kinshasa hospitals: Testimonies from women and their families. BMC Pregnancy Childbirth 11: 29.

41. Deboutte D, O' Dempsey T, Faragher B, Amuda Baba A (2016) Socioeconomic consequences of maternal death: A Qualitative study in Bunia, Democratic Republic of the Congo. Int J Womens Health Wellness 2: 027.

42. Punita A (2005) Maternal mortality-Indian scenario. Med J Armed Forces India 61: 214-215. 